\title{
QED? Not yet!
}

\author{
Can Yerebakan, MD
}

\footnotetext{
From the Department of Cardiovascular Surgery, Children's National Heart Institute, The George Washington University School of Medicine and Health Sciences, Washington, DC.

Disclosures: Author has nothing to disclose with regard to commercial support.

Received for publication Jan 19, 2018; accepted for publication Jan 30, 2018; available ahead of print Feb 28, 2018.

Address for reprints: Can Yerebakan, MD, Children's National Heart Institute, The George Washington University School of Medicine and Health Sciences, 111 Michigan Ave NW, Washington, DC 20010 (E-mail: cyerebakan@childrensnational.org).

J Thorac Cardiovasc Surg 2018;155:2565-6

$0022-5223 / \$ 36.00$

Copyright (C) 2018 by The American Association for Thoracic Surgery

https://doi.org/10.1016/j.jtcvs.2018.01.050
}

Anomalous aortic origin of a coronary artery (AAOCA) from the opposite sinus of Valsalva is a very rare congenital anomaly with a documented potentially devastating risk of sudden cardiac death in young individuals. Starting from the acknowledgement of the entity as a pathology, rather than just a normal anatomic variation, the journey toward an evidence-based treatment has been expedited during the last few years. ${ }^{1,2}$ Risk stratification of patients continues to remain a challenge, despite the effort to create a detailed description of guidelines. ${ }^{2,3}$

In this issue of the Journal, Nees and colleagues ${ }^{4}$ present $^{-}$ their retrospective review of 60 patients who underwent a repair exclusively for AAOCA in 2 affiliated institutions. The study, in line with other reports in the literature, offers enough reason to reconsider our surgical techniques to reduce morbidity in these patients. ${ }^{5,6}$ Even with a short median follow-up period of 1.6 years, Nees and colleagues ${ }^{4}$ emphasize 2 unfavorable surgical outcome features. First, a restenosis of the repaired coronary may occur even without positive stress testing findings, resulting in sudden cardiac death years after surgery. In this study, 2 patients required reoperation as a result of coronary stenosis at 3 months and 6 months after surgery. Second, aortic valve regurgitation has been observed to a mild and greater degree in $17 \%$ of the patients postoperatively within this short follow-up. One patient underwent reoperation for aortic regurgitation. In addition to this, supra-aortic stenosis was encountered in 1 patient, and a resolution of the preoperative symptoms could only be documented in $64 \%$ of the preoperatively symptomatic patients. Nees and colleagues ${ }^{4}$ are to be congratulated for their detailed analysis of a large cohort of the patients and particularly for attracting attention to these extremely important outcome measures. With the inherent limitations of a retrospective, single-center study with a short follow-up but also with a high number of patients, the messages of this evaluation are of great value, highlighting the previously mentioned aspects and leading us to reconsider the impression of apparently "excellent" surgical outcomes in repair of AAOCAs. We know that

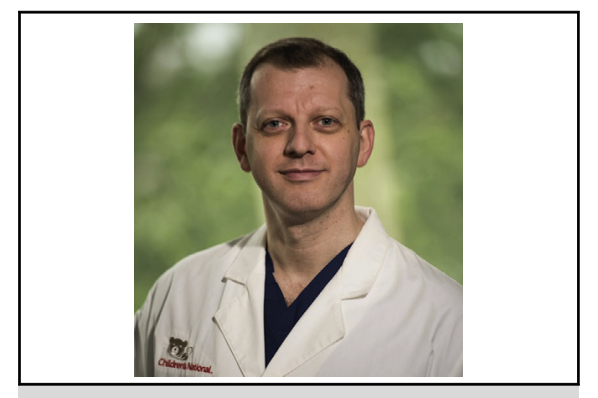

Can Yerebakan, MD

Central Message

The surgical treatment of an anomalous aortic origin of a coronary artery requires refinement in 3 aspects: indication, techniques, and overall outcome.

See Article page 2554.

patients after AAOCA repair will remain at risk for lifetime. A clear understanding of the reasons for suboptimal outcomes in some cases is missing. Preoperative evaluations, surgical practices, and follow-up protocols that have significant variations between institutions do not make problem solving easy. As necessary for every surgical patient, each institution will require standardized protocols to allow a smooth multidisciplinary approach to the treatment and follow-up of patients with AAOCA. We are urged to aim strictly for an excellent surgical repair with the least morbidity for these mostly young patients, particularly with current challenges of risk assessment.

There is a consensus among experts that anatomic features should determine the type of repair. For instance, recent reports have suggested that an unroofing of a short intramural course might not suffice to relieve the risk of a recurrent obstruction. ${ }^{7}$ A reimplantation therefore may be necessary if the coronary ostium cannot be moved beyond the intercoronary pillar to its home sinus. It is likely that similar experiences will be reported in the future, addressing different morphologic features with alternate surgical techniques. Furthermore, once longterm data arrive, we will probably learn more about the fate of the intimal readaptation sutures (suture line) around the coronary ostium with regard to the tendency toward scarring and calcification. Intimal disruption and readaptation, especially in smaller ostial openings, may expose patients to a higher risk for adverse cardiac events and an eventual revascularization requirement in the longer term. 
Less evidence exists in cases with an intramural course that is close to or even below the aortic valve commissure with regard to the technique of unroofing. Here, the definition of close remains unknown. Unroofing of an intramural coronary has the potential to distort the support of the aortic valve commissure with immediate proximity. Because the number of patients with new postoperative aortic regurgitation is not negligible in some studies, even without commissural takedown, the question has to be asked whether a resuspension of the aortic valve commissure needs to be performed in a standard manner regardless of the coronary proximity to the commissure. Preliminary data from my own center's experience indicate that a resuspension can be accomplished with no increased surgical risk but with superior aortic valve function in the medium-term follow-up relative to those patients without resuspension. ${ }^{8}$ Another disproportionate complication seems the occurrence of the postcardiotomy syndrome with probable pericardial effusion. $^{4,5}$ Surgical (pleuropericardial window) or medical (anti-inflammatory treatment) may reduce both the burden for our patients and rehospitalization rates.

We are still distant from adequately answering the question of whether today's surgical indications and repair techniques for AAOCA are effective to prevent sudden cardiac death with acceptable surgical morbidity and sufficient scientific evidence. It thus will not be sufficient to declare the surgery mere to be a safe approach in future to proceed with current practices. Prospective, multicenter studies with longer follow-up periods and standardized, detailed protocols throughout the management of this entity are more essential than ever.

\section{References}

1. Cheitlin MD, De Castro CM, McAllister HA. Sudden death as a complication of anomalous left coronary origin from the anterior sinus of Valsalva, a not-so-minor congenital anomaly. Circulation. 1974;50:780-7.

2. Brothers JA, Frommelt MA, Jaquiss RD, Myerburg RJ, Fraser CD Jr, Tweddell JS. Expert consensus guidelines: anomalous aortic origin of a coronary artery. $J$ Thorac Cardiovasc Surg. 2017;153:1440-57.

3. Van Hare GF, Ackerman MJ, Evangelista JK, Kovacs RJ, Myerburg RJ, Shafer KM, et al. Eligibility and disqualification recommendations for competitive athletes with cardiovascular abnormalities: task force 4: congenital heart disease: a scientific statement from the American Heart Association and American College of Cardiology. J Am Coll Cardiol. 2015;66:2372-84.

4. Nees SN, Flyer JN, Chelliah A, Dayton JD, Touchette L, Kalfa D, et al. Patients with anomalous aortic origin of the coronary artery remain at risk after surgical repair. J Thorac Cardiovasc Surg. 2018;155:2554-64.e3.

5. Wittlieb-Weber CA, Paridon SM, Gaynor JW, Spray TL, Weber DR, Brothers JA. Medium-term outcome after anomalous aortic origin of a coronary artery repair in a pediatric cohort. J Thorac Cardiovasc Surg. 2014;147:1580-6.

6. Brothers JA, McBride MG, Seliem MA, Marino BS, Tomlinson RS, Pampaloni $\mathrm{MH}$, et al. Evaluation of myocardial ischemia after surgical repair of anomalous aortic origin of a coronary artery in a series of pediatric patients. $J$ Am Coll Cardiol. 2007;50:2078-82.

7. Mery CM, De León LE, Molossi S, Sexson-Tejtel SK, Agrawal H, Krishnamurthy R, et al. Outcomes of surgical intervention for anomalous aortic origin of a coronary artery: a large contemporary prospective cohort study. $J$ Thorac Cardiovasc Surg. 2018;155:305-19.e4.

8. Yerebakan C, Ozturk M, Sinha L, Jonas RA, Sinha P. Complete unroofing of the intramural coronary artery for anomalous aortic origin of the coronary artery: the role of aortic commissural resuspension. Presented at: 96th Annual Meeting of The American Association for Thoracic Surgery; April 28-May 1, 2018; San Diego, CA. 